\title{
Continued Fractions of Tails of Hypergeometric Series
}

\section{Jonathan Michael Borwein, Kwok-Kwong Stephen Choi, and Wilfried Pigulla}

1. MOTIVATION. The tails of the Taylor series for many standard functions such as the arctangent and the logarithm can be expressed as continued fractions in a variety of ways. A surprising side effect is that some of these continued fractions provide a dramatic acceleration for the convergence of the underlying power series. These investigations were motivated by a surprising observation about Gregory's series. Gregory's series for $\pi$, truncated at 500,000 terms, gives to forty places

$$
4 \sum_{k=1}^{500.000} \frac{(-1)^{k-1}}{2 k-1}=3.14159 \underline{0} 6535897932404626433832 \underline{6} 9502884197 \ldots
$$

To one's initial surprise only the underlined digits are wrong (i.e, they differ from those of $\pi$ ). This is explained, ex post facto, by setting $N$ equal to one million in the following result:

Theorem 1. For an integer $N$ divisible by 4 the following asymptotic expansion holds:

$$
\begin{aligned}
\frac{\pi}{2}-2 \sum_{k=1}^{N / 2} \frac{(-1)^{k-1}}{2 k-1} & \sim \sum_{m=0}^{\infty} \frac{E_{2 m}}{N^{2 m+1}} \\
& =\frac{1}{N}-\frac{1}{N^{3}}+\frac{5}{N^{5}}-\frac{61}{N^{7}}+\cdots,
\end{aligned}
$$

where the numerators $1,-1,5,-61,1385,-50521, \ldots$ are the Euler numbers $E_{0}$, $E_{2}, E_{4}, E_{6}, E_{8}, E_{10}, \ldots$

The observation (1) came to the first author's attention in 1987. After verifying its truth numerically (which is much quicker today), it was an easy matter to generate a large number of the "errors" to high precision. The authors of [1] then recognized the sequence of errors in (1) as the Euler numbers - with the help of Sloane's Handbook of Integer Sequences. (The presumption that (1) is a form of Euler-Maclaurin summation is now formally verifiable for any fixed $N$ with the aid of MAPLE.) This allowed them to determine that (1) is equivalent to a set of identities between Bernoulli and Euler numbers that could with considerable effort have been established.

Secure in the knowledge that (1) held they found it easier, however, to use the Boole summation formula, which applies directly to alternating series, and Euler numbers (see [1]). Because $N$ was a power of ten, the asymptotic expansion was obvious on the computer screen. When one uses $N=10^{m}$ in (2) the errors will appear in the $m$ th place- until the Euler numbers grow too large. This is a good example of a phenomenon that really does not become apparent without working to reasonably high 
precision (Who recognizes $2,-2,10$ ?) and highlights the role of pattern recognition and hypothesis validation in experimental mathematics.

It was an amusing additional exercise to compute $\pi$ to 5,000 digits from (1). Indeed, taking $N=200,000$ and correcting using the first thousand even Euler numbers, Borwein and Limber [2] obtained 5,263 digits of $\pi$ (plus 12 guard digits). Thus, while the alternating Gregory series is very slowly convergent, the errors are highly predictable.

Although the connection is not immediately obvious, the present paper was stimulated by the third author's attempt to explain the phenomenon via continued fractions.

2. THREE CONTINUED FRACTION CLASSES. In this section we discuss three classes of continued fractions, namely, those of Euler, Gauss, and Perron.

Euler's continued fraction. Using the following notation for continued fractions,

$$
\frac{a_{1}}{b_{1}} \pm \frac{a_{2}}{b_{2}} \pm \frac{a_{3}}{b_{3}} \pm \cdots=\frac{a_{1}}{b_{1} \pm \frac{a_{2}}{b_{2} \pm \frac{a_{3}}{b_{3} \pm \ddots}}}
$$

identities such as

$$
a_{0}+a_{1}+a_{1} a_{2}+a_{1} a_{2} a_{3}+a_{1} a_{2} a_{3} a_{4}=a_{0}+\frac{a_{1}}{1}-\frac{a_{2}}{1+a_{2}}-\frac{a_{3}}{1+a_{3}}-\frac{a_{4}}{1+a_{4}}
$$

are easily verified symbolically. The general form

$$
\begin{aligned}
& a_{0}+a_{1}+a_{1} a_{2}+a_{1} a_{2} a_{3}+\cdots+a_{1} a_{2} a_{3} \cdots a_{N} \\
& =a_{0}+\frac{a_{1}}{1}-\frac{a_{2}}{1+a_{2}}-\frac{a_{3}}{1+a_{3}}-\cdots-\frac{a_{N}}{1+a_{N}}
\end{aligned}
$$

can then be obtained by substituting $a_{N}+a_{N} a_{N+1}$ for $a_{N}$ and checking that the shape of the right-hand side is preserved. This allows many series to be reexpressed as continued fractions. For example, taking $a_{0}=0, a_{1}=z, a_{2}=-z^{2} / 3, a_{3}=-3 z^{2} / 5, \ldots$ and using the Taylor expansion

$$
\arctan z=z-\frac{z^{3}}{3}+\frac{z^{5}}{5}-\frac{z^{7}}{7}+\frac{z^{9}}{9}-\cdots,
$$

we obtain by letting $N \rightarrow \infty$ in (3) the continued fraction for the arctangent due to Euler:

$$
\arctan z=\frac{z}{1}+\frac{z^{2}}{3-z^{2}}+\frac{9 z^{2}}{5-3 z^{2}}+\frac{25 z^{2}}{7-5 z^{2}}+\cdots
$$

When $z=1$, this becomes the first infinite continued fraction, given by Lord Brouncker $(1620-1684)$ :

$$
\frac{4}{\pi}=1+\frac{1}{2}+\frac{9}{2}+\frac{25}{2}+\frac{49}{2}+\cdots
$$

If we let $a_{0}=\sum_{1}^{N} b_{k}$ be the initial segment of a similar series, we can use (3) to replace the remaining terms with a continued fraction. For example, if we put

$$
a_{0}=\sum_{n=1}^{N} \frac{(-1)^{n-1} z^{2 n-1}}{2 n-1}, \quad a_{1}=\frac{(-1)^{N} z^{2 N+1}}{2 N+1},
$$




$$
a_{2}=-\frac{2 N+1}{2 N+3} z^{2}, \quad a_{3}=-\frac{2 N+3}{2 N+5} z^{2}, \ldots,
$$

then we get

$$
\begin{aligned}
\arctan z= & \sum_{n=1}^{N}(-1)^{n-1} \frac{z^{2 n-1}}{2 n-1}+\frac{(-1)^{N} z^{2 N+1}}{2 N+1}+\frac{(2 N+1)^{2} z^{2}}{(2 N+3)-(2 N+1) z^{2}} \\
& +\frac{(2 N+3)^{2} z^{2}}{(2 N+5)-(2 N+3) z^{2}}+\frac{(2 N+5)^{2} z^{2}}{(2 N+7)-(2 N+5) z^{2}}+\cdots
\end{aligned}
$$

Gauss's continued fraction. A rich vein lies in Gauss's continued fraction for the ratio of two hypergeometric functions $\mathrm{F}(a, b+1 ; c+1 ; z) / \mathrm{F}(a, b ; c ; z)$ (see [5]). Recall that within its radius of convergence the Gaussian hypergeometric function is defined by

$$
\begin{aligned}
\mathrm{F}(a, b ; c ; z)=1 & +\frac{a b}{c} z+\frac{a(a+1) b(b+1)}{2 ! c(c+1)} z^{2} \\
& +\frac{a(a+1)(a+2) b(b+1)(b+2)}{3 ! c(c+1)(c+2)} z^{3}+\cdots
\end{aligned}
$$

The general continued fraction is developed by a reworking of the contiguity relation

$$
\mathrm{F}(a, b ; c ; z)=\mathrm{F}(a, b+1 ; c+1 ; z)-\frac{a(c-b)}{c(c+1)} z \mathrm{~F}(a+1, b+1 ; c+2 ; z)
$$

and at least formally is quite easy to derive. Convergence and convergence estimates are more delicate. We therefore have

$$
\frac{\mathrm{F}(a, b+1 ; c+1 ; z)}{\mathrm{F}(a, b ; c ; z)}=\left(1-\frac{a(c-b)}{c(c+1)} z \frac{\mathrm{F}(a+1, b+1 ; c+2 ; z)}{\mathrm{F}(a, b+1 ; c+1 ; z)}\right)^{-1},
$$

which leads to the recursive process for the continued fraction. By taking $b=0$ and replacing $c$ with $c-1$, this process yields in the limit

$$
\mathrm{F}(a, 1 ; c ; z)=\frac{1}{1}-\frac{a_{1} z}{1}-\frac{a_{2} z}{1}-\frac{a_{3} z}{1}-\cdots,
$$

which is the case of present interest. Here

$$
a_{2 l+1}=\frac{(a+l)(c-1+l)}{(c+2 l-1)(c+2 l)}, \quad a_{2 l+2}=\frac{(l+1)(c-a+l)}{(c+2 l)(c+2 l+1)}
$$

for $l=0,1, \ldots$ We also let

$$
F_{M}(a, 1 ; c ; z)=\frac{1}{1}-\frac{a_{1} z}{1}-\frac{a_{2} z}{1}-\cdots \frac{a_{M-1} z}{1}
$$

denote the $M$ th convergent of the continued fraction to $F(a, 1 ; c ; z)$.

It is well known and easy to verify that $\log (1+z)=z \mathrm{~F}(1,1 ; 2 ;-z)$. It is a pleasant surprise to discover that

$$
\log (1+z)-z=-\frac{1}{2} z^{2} \mathrm{~F}(2.1 ; 3 ;-z)
$$


and

$$
\log (1+z)-z+\frac{1}{2} z^{2}=\frac{1}{3} z^{3} \mathrm{~F}(3,1 ; 4 ;-z)
$$

and then to conjecture that

$$
\log (1+z)+\sum_{n=1}^{N-1} \frac{(-1)^{n} z^{n}}{n}=-\frac{(-1)^{N} z^{N}}{N} \mathrm{~F}(N, 1 ; N+1 ;-z)
$$

This is straightforward to establish for the first few cases and then confirm rigorously. As always, a formula for the logarithm leads correspondingly to one for the arctangent:

$$
\arctan z-\sum_{n=0}^{N-1} \frac{(-1)^{n} z^{2 n+1}}{2 n+1}=\frac{(-1)^{N} z^{2 N+1}}{2 N+1} \mathrm{~F}\left(N+\frac{1}{2}, 1 ; N+\frac{3}{2} ;-z^{2}\right) .
$$
as

Happily, in both cases (8) is applicable - as it is for a variety of other functions such

$$
\log \left(\frac{1+z}{1-z}\right), \quad(1+z)^{k}, \quad \int_{0}^{z}\left(1+t^{n}\right)^{-1} d t=z \mathrm{~F}\left(\frac{1}{n}, 1 ; 1+\frac{1}{n} ;-z^{n}\right) .
$$

Note that this last function recaptures $\log (1+z)$ and $\arctan z$ for $n=1$ and 2 , respectively.

We next give the explicit continued fractions for (9) and (10).

Theorem 2. Gauss's continued fractions for (9) and (10) are

$$
\begin{aligned}
\log (1+z)+\sum_{n=1}^{N-1} \frac{(-1)^{n} z^{n}}{n} & \\
& =\frac{(-1)^{N+1} z^{N}}{N}+\frac{N^{2} z}{N+1}+\frac{1^{2} z}{N+2}+\frac{(N+1)^{2} z}{N+3}+\frac{2^{2} z}{N+4}+\cdots
\end{aligned}
$$

and

$$
\begin{aligned}
\arctan z & -\sum_{n=0}^{N-1} \frac{(-1)^{n} z^{2 n+1}}{2 n+1} \\
& =\frac{(-1)^{N} z^{2 N+1}}{2 N+1}+\frac{(2 N+1)^{2} z^{2}}{2 N+3}+\frac{2^{2} z^{2}}{2 N+5}+\frac{(2 N+3)^{2} z^{2}}{2 N+7}+\frac{4^{2} z^{2}}{2 N+9}+\cdots
\end{aligned}
$$

Suppose that we return to Gregory's series but add a few terms of the continued fraction for (10). One observes numerically that, if the results are for $N=500,000$, then adding only six terms of the continued fraction has the effect of increasing the precision by forty digits.

Example 3. Let

$$
E_{1}(N, M, z):=\log (1+z)-\left(-\sum_{n=1}^{N} \frac{(-z)^{n}}{n}-\frac{(-z)^{N+1}}{N+1} F_{M}(N+1,1 ; N+2 ;-z)\right)
$$


and

$$
\begin{aligned}
& E_{2}(N, M, z) \\
& \quad:=\arctan z-\left(\sum_{n=0}^{N-1} \frac{(-1)^{n} z^{2 n+1}}{2 n+1}+\frac{(-1)^{N} z^{2 N+1}}{2 N+1} \mathrm{~F}_{M}\left(N+\frac{1}{2}, 1 ; N+\frac{3}{2} ;-z^{2}\right)\right) .
\end{aligned}
$$

Then $E_{1}(N, M, z)$ and $E_{2}(N, M, z)$ measure the precision of the approximations to $\log (1+z)$ and $\arctan z$, respectively, obtained by initially computing the first $N$ terms of their Taylor series and then adding $M$ terms of their continued fractions. Tables 1 , 2,3 , and 4 record those data for the approximations to $\log (1.9), \log (2), \arctan (1)$, and $\arctan (1 / 2)+\arctan (1 / 5)+\arctan (1 / 8)$, respectively. Note that

$$
\frac{\pi}{4}=\arctan \left(\frac{1}{2}\right)+\arctan \left(\frac{1}{5}\right)+\arctan \left(\frac{1}{8}\right)
$$

is a formula of Machin-type used by Johann Dase in 1844 to compute 205 digits of $\pi$

\begin{tabular}{|c|c|c|c|c|c|}
\hline & & $5 \times 10$ & $5 \times 10^{2}$ & $5 \times 10^{3}$ & $5 \times 10^{4}$ \\
\hline & () & $0.48 \times 10^{-4}$ & $0.13 \times 10^{-2.5}$ & $0.15 \times 10^{-232}$ & $0.13 \times 10^{-2242}$ \\
\hline & 1 & $0.43 \times 10^{-4}$ & $0.11 \times 10^{-25}$ & $0.14 \times 10^{-232}$ & $0.11 \times 10^{-29 y}$ \\
\hline & 2 & $0.40 \times 10^{-8}$ & $0.11 \times 10^{-31}$ & $0.14 \times 10^{-240}$ & $0.11 \times 10^{-23112}$ \\
\hline \multirow[t]{4}{*}{$M$} & 3 & $0.34 \times 10^{-x}$ & $1.00 \times 10^{-32}$ & $0.12 \times 10^{-241}$ & $0.10 \times 10^{-2312}$ \\
\hline & 4 & $0.12 \times 10^{-11}$ & $0.40 \times 10^{-37}$ & $0.50 \times 10^{-248}$ & $0.41 \times 10^{-2312}$ \\
\hline & 5 & $0.10 \times 10^{-11}$ & $0.35 \times 10^{-37}$ & $0.45 \times 10^{-248}$ & $0.37 \times 10^{-2312}$ \\
\hline & 6 & $0.78 \times 10^{-15}$ & $0.31 \times 10^{-42}$ & $0.40 \times 10^{-255}$ & $0.33 \times 10^{-2321}$ \\
\hline
\end{tabular}
in his head.

Table 1. Error $\left|E_{1}(N . M .0 .9)\right|$ for $N=5 \times 10^{k}(1 \leq k \leq 4)$ and $0 \leq M \leq 6$.

Table 2. Error $\left|E_{\mid}(N, M, 1)\right|$ for $N=5 \times 10^{k}(1 \leq k \leq 6)$ and $0 \leq M \leq 6$.

\begin{tabular}{c|ccccccc}
\hline & $5 \times 10$ & $5 \times 10^{2}$ & $5 \times 10^{3}$ & $5 \times 10^{4}$ & $5 \times 10^{5}$ & $5 \times 10^{6}$ \\
\hline & 0 & $0.99 \times 10^{-2}$ & $1.00 \times 10^{-3}$ & $1.00 \times 10^{-4}$ & $1.00 \times 10^{-5}$ & $1.00 \times 10^{-6}$ & $1.00 \times 10^{-7}$ \\
& 1 & $0.97 \times 10^{-2}$ & $1.00 \times 10^{-3}$ & $1.00 \times 10^{-4}$ & $1.00 \times 10^{-5}$ & $1.00 \times 10^{-6}$ & $1.00 \times 10^{-7}$ \\
& 2 & $0.91 \times 10^{-6}$ & $1.00 \times 10^{-9}$ & $1.00 \times 10^{-12}$ & $1.00 \times 10^{-15}$ & $1.00 \times 10^{-18}$ & $1.00 \times 10^{-21}$ \\
\hline & 3 & $0.86 \times 10^{-6}$ & $1.00 \times 10^{-9}$ & $1.00 \times 10^{-12}$ & $1.00 \times 10^{-1.5}$ & $1.00 \times 10^{-18}$ & $1.00 \times 10^{-21}$ \\
& 4 & $0.31 \times 10^{-9}$ & $0.39 \times 10^{-14}$ & $0.40 \times 10^{-19}$ & $0.40 \times 10^{-24}$ & $0.40 \times 10^{-29}$ & $0.40 \times 10^{-34}$ \\
& 5 & $0.28 \times 10^{-4}$ & $0.39 \times 10^{-14}$ & $0.40 \times 10^{-19}$ & $0.40 \times 10^{-24}$ & $0.40 \times 10^{-29}$ & $0.40 \times 10^{-34}$ \\
& 6 & $0.22 \times 10^{-12}$ & $0.34 \times 10^{-19}$ & $0.36 \times 10^{-26}$ & $0.36 \times 10^{-3.3}$ & $0.36 \times 10^{-40}$ & $0.36 \times 10^{-47}$ \\
\hline
\end{tabular}

Table 3. Error $\left|E_{2}(N, M, 1)\right|$ for $N=5 \times 10^{k}(1 \leq k \leq 0)$ and $0 \leq M \leq 0$.

\begin{tabular}{cc|cccccc}
\hline & $5 \times 10^{2}$ & $5 \times 10^{2}$ & $5 \times 10^{3}$ & $5 \times 10^{+}$ & $5 \times 10^{5}$ & $5 \times 10^{6}$ \\
\hline \multirow{4}{*}{$M$} & 0 & $0.50 \times 10^{-2}$ & $0.50 \times 10^{-3}$ & $0.50 \times 10^{-4}$ & $0.50 \times 10^{-5}$ & $0.50 \times 10^{-6}$ & $0.50 \times 10^{-7}$ \\
& 1 & $0.49 \times 10^{-2}$ & $0.50 \times 10^{-3}$ & $0.50 \times 10^{-4}$ & $0.50 \times 10^{-5}$ & $0.50 \times 10^{-6}$ & $0.50 \times 10^{-7}$ \\
& 2 & $0.47 \times 10^{-6}$ & $0.50 \times 10^{-9}$ & $0.50 \times 10^{-12}$ & $0.50 \times 10^{-1.5}$ & $0.50 \times 10^{-18}$ & $0.50 \times 10^{-21}$ \\
& 3 & $0.44 \times 10^{-6}$ & $0.49 \times 10^{-9}$ & $0.50 \times 10^{-12}$ & $0.50 \times 10^{-15}$ & $0.50 \times 10^{-18}$ & $0.50 \times 10^{-21}$ \\
& 4 & $0.16 \times 10^{-9}$ & $0.20 \times 10^{-14}$ & $0.20 \times 10^{-19}$ & $0.20 \times 10^{-24}$ & $0.20 \times 10^{-29}$ & $0.20 \times 10^{-34}$ \\
& 5 & $0.15 \times 10^{-4}$ & $0.19 \times 10^{-14}$ & $0.20 \times 10^{-19}$ & $0.20 \times 10^{-24}$ & $0.20 \times 10^{-29}$ & $0.20 \times 10^{-34}$ \\
6 & $0.12 \times 10^{-12}$ & $0.17 \times 10^{-19}$ & $0.18 \times 10^{-26}$ & $0.18 \times 10^{-3.3}$ & $0.18 \times 10^{-40}$ & $0.18 \times 10^{-47}$ \\
\hline
\end{tabular}


Table 4. Error $\left|E_{2}(N+1, M, 1 / 2)+E_{2}(N+1, M, 1 / 5)+E_{2}(N+1, M, 1 / 8)\right|$ for $N=5 \times 10^{k}(1 \leq k \leq 2)$ and $0 \leq M \leq 6$.

\begin{tabular}{cc|cc}
\hline & & $5 \times 10$ & $5 \times 10^{2}$ \\
\hline & 0 & $0.31 \times 10^{-32}$ & $0.37 \times 10^{-304}$ \\
& 1 & $0.19 \times 10^{-33}$ & $0.23 \times 10^{-305}$ \\
& 2 & $0.11 \times 10^{-37}$ & $0.15 \times 10^{-311}$ \\
$M$ & 3 & $0.26 \times 10^{-38}$ & $0.37 \times 10^{-312}$ \\
& 4 & $0.56 \times 10^{-42}$ & $0.92 \times 10^{-318}$ \\
& 5 & $0.13 \times 10^{-42}$ & $0.23 \times 10^{-318}$ \\
& 6 & $0.59 \times 10^{-46}$ & $0.13 \times 10^{-323}$ \\
\hline
\end{tabular}

After some further numerical experimentation it becomes clear that for large $a$ and $c$ the continued fraction $\mathrm{F}(a, 1, c ; z)$ is rapidly convergent. Indeed, the rough rate is apparent. This is part of the content of the next theorem:

Theorem 4. Suppose that $2 \leq a, a+1 \leq c \leq 2 a$, and $M \geq 2$. If $-1 \leq z<0$, then

$$
\begin{aligned}
& \left|\mathrm{F}(a, 1 ; c ; z)-\mathrm{F}_{M}(a, 1 ; c ; z)\right| \\
& \quad \leq \frac{\Gamma(n+1)(n+a) \Gamma(n+c-a) \Gamma(a) \Gamma(c)}{\Gamma(n+a) \Gamma(n+c) a \Gamma(c-a)}\left(\frac{2 a}{(c-2)\left(1-\frac{2}{z}\right)+(2 a-c)}\right)^{M},
\end{aligned}
$$

where $n=[M / 2]$ and $\mathrm{F}_{M}(a, 1 ; c ; z)$ is the Mth convergent of the continued fraction to $\mathrm{F}(a, 1, c ; z)$.

Proof. Recall that Gauss's continued fraction for $\mathrm{F}(a, 1 ; c ; z)$ is

$$
\mathrm{F}(a, 1 ; c ; z)=\frac{1}{1}-\frac{a_{1} z}{1}-\frac{a_{2} z}{1}-\frac{a_{3} z}{1}-\cdots,
$$

where

$$
a_{2 l+1}=\frac{(a+l)(c-1+l)}{(c+2 l-1)(c+2 l)}, \quad a_{2 l+2}=\frac{(l+1)(c-a+l)}{(c+2 l)(c+2 l+1)}
$$

for $l=0,1, \ldots$ Let

$$
\frac{A_{n}(z)}{B_{n}(z)}=\frac{1}{1}-\frac{a_{1} z}{1}-\frac{a_{2} z}{1}-\cdots-\frac{a_{n-1} z}{1}=\mathrm{F}_{n}(a, 1 ; c ; z)
$$

be the $n$th convergent of the continued fraction. It can be proved by induction that $A_{1}(z)=A_{2}(z)=B_{1}(z)=1$ and $B_{2}(z)=1-a_{1} z$, while

$$
A_{k}(z)=A_{k-1}(z)-a_{k-1} z A_{k-2}(z),
$$

and

$$
B_{k}(z)=B_{k-1}(z)-a_{k-1} z B_{k-2}(z)
$$

when $k \geq 3$. Hence for $k \geq 2$ we have

$$
A_{k}(z) B_{k-1}(z)-A_{k-1}(z) B_{k}(z)=a_{1} \cdots a_{k-1} z^{k-1} .
$$


Using the estimation in Theorem 8.9 of [3], we find that, if $a_{i}>0$ for all $i$, then

$$
\left|\mathrm{F}(a, 1 ; c ; z)-\frac{A_{n}(z)}{B_{n}(z)}\right| \leq\left|\frac{A_{n}(z)}{B_{n}(z)}-\frac{A_{n-1}(z)}{B_{n-1}(z)}\right|=\left|\frac{a_{1} \cdots a_{n-1} z^{n-1}}{B_{n}(z) B_{n-1}(z)}\right| .
$$

It can be shown that the $B_{n}(z)$ are hypergeometric polynomials (see [5]). Explicitly,

$$
B_{2 k}(z)=F(-k, 1-a-k, 2-c-2 k ; z)
$$

and

$$
B_{2 k+1}(z)=F(-k,-a-k, 1-c-2 k ; z) .
$$

We then appeal to estimates for hypergeometric polynomials to complete the argument. (A more detailed proof can be found in the associated technical report on the CECM preprint server for 2003 at http://eprints.cecm.sfu.ca/archive/ 00000039/.)

In [5] one can find listed many explicit continued fractions that can be derived from either Gauss's continued fraction or its limiting cases. These include the expansions of the exponential function, the hyperbolic tangent, the tangent, and various less elementary functions. One especially attractive fraction is that for $J_{n-1}(z) / J_{n}(z)$ and $I_{n-1}(z) / I_{n}(z)$, where $J$ and $I$ are Bessel functions of the first kind. In particular,

$$
\frac{J_{n-1}(2 z)}{J_{n}(2 z)}=\frac{n}{z}-\frac{\frac{z}{(n+1)}}{1}-\frac{\frac{z^{2}}{(n+1)(n+2)}}{1}-\frac{\frac{z^{2}}{(n+2)(n+3)}}{1}-\cdots
$$

Setting $z=i$ and $n=1,(13)$ leads to the very beautiful continued fraction

$$
\frac{I_{1}(2)}{I_{0}(2)}=\frac{1}{1}+\frac{1}{2}+\frac{1}{3}+\frac{1}{4}+\cdots
$$

since $I_{n}(z)=e^{-n \pi i / 2} J_{n}\left(z e^{\pi i / 2}\right)$. In general, arithmetic simple continued fractions correspond to such ratios.

An example of a more complicated situation is:

$$
\frac{(2 z)^{2 N+1} \mathrm{~F}\left(N+\frac{1}{2}, \frac{1}{2} ; N+\frac{3}{2} ; z^{2}\right)}{(N+1)\left(\begin{array}{c}
2 N+2 \\
N+1
\end{array}\right) \mathrm{F}\left(\frac{1}{2},-\frac{1}{2} ; \frac{1}{2} ; z^{2}\right)}=\frac{\arcsin z}{\sqrt{1-z^{2}}}-\sigma_{2 N}(z)
$$

where $\sigma_{2 N}$ is the $2 N$ th Taylor polynomial for $\arcsin z / \sqrt{1-z^{2}}$. Only for $N=0$ is this precisely of the form of Gauss's continued fraction.

Perron's continued fraction. Another continued fraction expansion is based on Stieltjes work on the moment problem (see Perron [4]). In [4, vol. 2, p. 18] one finds the lovely continued fraction

$$
\frac{1}{z^{\mu}} \int_{0}^{z} \frac{t^{\mu}}{1+t} d t=\frac{z}{\mu+1}+\frac{(\mu+1)^{2} z}{(\mu+2)-(\mu+1) z}+\frac{(\mu+2)^{2} z}{(\mu+3)-(\mu+2) z}+\cdots,
$$


which is valid when $\mu>-1$ and $-1<z \leq 1$. We can obtain this as a consequence of Euler's continued fraction if we write

$$
\frac{1}{z^{\mu}} \int_{0}^{z} \frac{t^{\mu}}{1+t} d t=\frac{z}{\mu+1}-\frac{z^{2}}{\mu+2}+\frac{z^{3}}{\mu+3}-\frac{z^{4}}{\mu+4}+\cdots
$$

and observe that (15) follows from (3) in the limit (here $a_{0}=0, a_{1}=z /(\mu+1)$, $a_{n}=(-1)^{n+1} z(\mu+n-1) /(\mu+n)$ for $\left.n=2,3, \ldots\right)$.

Since

$$
\frac{z^{\mu+1}}{\mu+1} \mathrm{~F}(\mu+1,1 ; \mu+2 ;-z)=\int_{0}^{z} \frac{t^{\mu}}{1+t} d t
$$

and

$$
\frac{z^{2 \mu+1}}{2 \mu+1} \mathrm{~F}\left(\mu+\frac{1}{2}, 1 ; \mu+\frac{3}{2} ;-z^{2}\right)=\int_{0}^{z} \frac{t^{2 \mu}}{1+t^{2}} d t
$$

when $\mu>0$, on examining (9) and (10) we see that this is immediately applicable to provide Euler continued fractions for the tail of the logarithm and arctangent series. To be precise, we obtain:

Theorem 5. Perron's continued fractions for (9) and (10) are:

$$
\begin{aligned}
\log (1 & +z)+\sum_{n=1}^{N-1} \frac{(-1)^{n} z^{n}}{n} \\
& =\frac{(-1)^{N+1} z^{N}}{N}+\frac{N^{2} z}{(N+1)-N z}+\frac{(N+1)^{2} z}{(N+2)-(N+1) z}+\cdots
\end{aligned}
$$

and

$$
\begin{aligned}
& \arctan (z)-\sum_{n=0}^{N-1} \frac{(-1)^{n} z^{2 n+1}}{2 n+1} \\
& \quad=\frac{(-1)^{N} z^{2 N+1}}{2 N+1}+\frac{(2 N+1)^{2} z^{2}}{(2 N+3)-(2 N+1) z^{2}}+\frac{(2 N+3)^{2} z^{2}}{(2 N+5)-(2 N+3) z}+\cdots
\end{aligned}
$$

Moreover, whereas the Gauss and Euler/Perron continued fractions obtained are quite distinct, we note the agreement of (19) with (5). Indeed, as we have seen, Theorem 5 coincides with a special case of (3).

ACKNOWLEDGMENT. This work was supported by NSERC and by the Canada Research Chair Programme.

\section{REFERENCES}

1. J. Borwein, P. Borwein, and K. Dilcher, Pi, Euler numbers and asymptotic expansions, this MONTHLY 96 (1989) 681-687.

2. J. Borwein and M. Limber, Maple as a high precision calculator, Maple News Letter 8 (1992) 39-44; also available at ww. cecm.sfu.ca/preprints/1998pp.html.

3. W. Jones and W. Thron, Comtimued Fractions: Analytic Theory and Applications, Encyclopedia of Mathematics and Its Applications, vol. 11, Addison-Wesley, Reading, MA, 1980.

4. O. Perron, Die Lehre von den Kettenbrïchen. Chelsea, New York, 1950.

5. H. Wall, Analytic Theory of Continued Fractions, Chelsea, New York. 1948. 
JONATHAN BORWEIN was Shrum Professor of Science and founding director of the Centre for Experimental and Constructive Mathematics at Simon Fraser University (1993-2003). In 2004, he (re-)joined the Faculty of Computer Science at Dalhousie as a Canada Research Chair in Distributed and Collaborative Research. Born in St. Andrews in 1951. he received his D. Phil. from Oxford in 1974, as a Rhodes Scholar. Prior to joining SFU in 1993, he worked at Dalhousie (1974-91). Carnegie-Mellon (1980-82), and Waterloo (199193). His awards include the Chauvenet Prize (1993). Fellowship in the Royal Society of Canada (1994). in the American Association for the Advancement of Science (2002), and foreign membership in the Bulgarian Academy of Sciences (2003). Dr. Borwein is governor at large of the MAA (2004-07) and a past president of the Canadian Mathematical Society $(200(0)-02)$. His interests span pure (analysis), applied (optimization). computational (numerical and computational analysis) mathematics, and high performance computing. He has authored ten books-most recently two on experimental mathematics (www.expmath.info) and a monograph on variational analysis-as well as over 250 journal articles.

Faculty of Computer Science, Dalhomsie University, 6050 University Alenue, Halifax Nola Scotia, Canada B3H IW5

jborwein@@cs.dal.c'a

STEPHEN CHOI was born in Hong Kong and obtained his B.Sc. (1988) and M. Phil. (1991) from the University of Hong Kong. Under the supervision of Jeffrey Vaaler, he received his Ph.D. in mathematics from the University of Texas at Austin in 1996. In 2000, he joined Simon Fraser University following postdoctoral fellowships at the Institute for Advanced Studies, the Pacific Institute for the Mathematical Sciences (UBC and SFU), and the University of Hong Kong. His research interests lie in studying Diophantine equations, Diophantine approximation, and the merit factor of binary sequences.

CECM, Department of Mathematics, Simon Fraser University, Bamaby B.C.. Canada V5A IS6

kkchoi@cecm.sfitca

WILFRIED PIGULLA is a retired civil servant and amateur mathematician who lives in Germany. 